\title{
An Explorative Study on Major Stressors and Its Connection to Depression and Chronic Stress among German Elite Athletes
}

\author{
Insa Nixdorf, Raphael Frank, Jürgen Beckmann \\ Chair of Sport Psychology, Technische Universität München, Munich, Germany \\ Email: insa.nixdorf@tum.de
}

Received 12 August 2015; accepted 15 November 2015; published 18 November 2015

Copyright $@ 2015$ by authors and Scientific Research Publishing Inc.

This work is licensed under the Creative Commons Attribution International License (CC BY).

http://creativecommons.org/licenses/by/4.0/

\section{cC) (i) Open Access}

\begin{abstract}
Although research shows that chronic stress and specific stressful events (e.g. injury, failure) are associated with depressive symptoms in athletes, connections between chronic, major stressors and depressive symptoms are yet unknown. Therefore, the goal of the study was to gain new findings between major stressors and their relations to depressive symptoms and chronic stress. $A$ total of 134 elite athletes provided data in an online survey. In addition to quantitative measurements (current depressive symptoms, and level of chronic stress), possible stressors were assessed qualitatively with an open ended question. By using content analysis to explore stressors, three different types of stressors were categorized: Double burden, sport specific demands, and conditions. Further statistical analysis found these stressors to be associated with dropout intentions, depression and chronic stress. Athletes, who reported sport specific demands (such as high training loads) as major stressors indicated higher levels of chronic stress and depressive symptoms than athletes without major stressors. Further research investigating sources of stress and its association to depressive syndromes in athletes could benefit from considering sport specific factors.
\end{abstract}

\section{Keywords}

Sources of Stress, Athlete, Depression

\section{Introduction}

Stress is a major determinant in the development of depression in the general population (Hammen, Kim, Eberhart, \& Brennan, 2009; Lee, Jeong, Kwak, \& Park, 2010; Monroe \& Reid, 2009). Regarding the population of athletes in elite sports, stress is also discussed in relation to depressive syndromes according to Puffer \& 
McShane (1992). Recent results (Nixdorf, Frank, Hautzinger, \& Beckmann, 2013; Schaal et al., 2011) indicate that prevalence rates between $4 \%$ and $15 \%$ in elite athletes seem comparable to the general population in Europe (cf. Frank, Nixdorf, \& Beckmann, 2013). Due to the profound prevalence rates, further knowledge on specific connections between depression and stress in athletes is needed.

Stress can be characterized as being either acute or chronic. "Participating in competitive sports often places the athlete under intense physical, psychological, and emotional demands" (Crocker \& Graham, 1995: p. 325). And indeed, whether one takes into consideration the important tournaments and potential sporting injuries (acute stress) or the frequency of tournaments and training sessions (chronic stress), the life of an athlete can be regarded as "stressful". Nixdorf et al. (2013) found support for this assumption, indicating high associations between chronic stress and depressive syndromes, as well as between different coping strategies and depressive syndromes in elite athletes. But which are the main stressors, connected to experiences of chronic stress and depressive syndromes?

Sources of chronic stress can be located in the personal environment of an athlete as well as in the specific sport discipline that the athlete is competing in. Research on stressors in the context of sports shows that stressors can be found in the competitive surrounding as well as in the organization where an athlete is located in (Hanton, Fletcher, \& Coughlan, 2005). Organizational stressors, representing the environmental demands associated primarily and directly with the organization in which an individual was operating in, were shown to be multiple facetted with dimensions such as interpersonal demands or stress due to roles within the sport organization (see Fletcher, Hanton, Mellalieu, \& Neil, 2012). But besides stressors that are clearly related to the sport organization or the competitive nature of elite sports, athletes are also exposed to stressors such as job insecurity or difficulties balancing sport, study commitments (Noblet \& Gifford, 2002) and the physical demand of training (Gould, Jackson, \& Finch, 1993). Regarding the location of major stressors possibly connected to depressive syndromes in athletes, important stressors might be located within the sport organization, as well as within private challenges. Therefore, the contribution of specific factors in these domains needs further qualification.

Regarding which factors are associated with depressive symptoms, few articles provide information on specific triggers such as injuries (e.g. Appaneal, Levine, Perna, \& Roh, 2009; Leddy, Lambert, \& Ogles, 1994) and overtraining (Armstrong \& VanHeest, 2002; Meeusen et al., 2013; O’Connor, Morgan, Raglin, Barksdale, \& Kalin, 1989). The impact of chronic stress is mainly put in context with the overtraining syndrome (e.g. Kellmann, 2010; Lehmann, Gastmann, Keizer, \& Steinacker, 1999). Athletes undergoing a strenuous training schedule can develop a significant decrease in performance associated with systemic symptoms or signs, which are also shown to be associated with other negative phenomena such as burnout (Lemyre, Roberts, \& Stray-Gundersen, 2007). According to Angeli, Minetto, Dovio, \& Paccotti (2004), this is a stress-related condition that consists of alteration of physiological functions and adaptation to performance, impairment of psychological processing, immunological dysfunction and biochemical abnormalities. The other sources of chronic stress which are relevant for depression in athletes are still unknown. There is little research regarding major stressors contributing to experiences of chronic stress in athletes (e.g., cohesion of teams/training partners, pressure from supportive surrounding etc.). In addition, measurement of psychological influences is mostly based on the ascertainment of questionnaires. Whereas a great benefit of standardized questionnaires is their reliability and validity, individual aspects are usually neglected and a broader range of sport-related information that could stimulate the generation of new hypothesis is missed.

Summarizing the above raised questions, there is a lack of knowledge on whether and which (sport related) stressors have the potential to become relevant for depression and possible dropout in elite athletes. Consequently, the present study focuses on this matter by giving the questioned athletes the opportunity to rise to speak and name major stressors. Therefore, aim of the study was (1) to explore sport-relevant major stressors for elite athletes and (2) identify possible connections between these stressors and depressive syndromes as well as with chronic stress. Through this explorative approach new hypothesis and further assumptions regarding mechanisms for chronic stress and depression in elite athletes should be gathered. Assessment of merely major stressors was operationalized by asking elite athletes to state stressors related to a possible dropout of competitive sports. The answers were explored in an open, bottom up approach leading to a categorization of major stressors. As existing categorizations of stressors (e.g. Hanton et al., 2005) in elite athletes might be too narrow for the topic of depression this open and explorative approach was used. In addition, athlete's depressive symptoms and levels of chronic stress were assessed in order to test possible connections to the categorized stressors. 


\section{Method}

\subsection{Participants}

A total of 134 German elite athletes $\left(M_{\mathrm{age}}=21.66 ; S D=4.60\right.$; age range: $\left.15-37\right)$ were included $^{1}$. Of originally 162 participating athletes 28 athletes were excluded since they did not meet criteria for being an elite athlete. The decision about level of expertise was based on level of performance, age, hours of exercise per week, and number of competitions per season, with level of performance being the main criterion. Athletes in the national squad were automatically designated as elite athletes. Athletes in the first national league in handball, cycling, and volleyball and in the first and second national league in soccer were also designated as elite athletes. To ensure participant's adequate understanding and prevent inappropriate data, only complete cases (incomplete cases: $n=60$ ) and cases meeting honesty criteria (nonplausible answers, response set, etc.: $n=8$ ) were originally included. The final sample consisted of 56 female $\left(M_{\text {age }}=22.16 ; S D=4.75\right.$; age range: 15 - 33) and 78 male ( $M_{\text {age }}$ $=21.29 ; S D=4.48$; age range: $16-37)$ elite athletes. Sports included athletics $(n=2)$, badminton $(n=3)$, beach volleyball $(n=3)$, canoeing $(n=19)$, cycling $(n=38)$, golf $(n=4)$, handball $(n=15)$, hockey $(n=2)$, ice hockey $(n=1)$, ice running $(n=5)$, modern pentathlon $(n=1)$, rugby $(n=12)$, snowboarding $(n=2)$, soccer $(n=20)$, swimming $(n=1)$, triathlon $(n=2)$, volleyball $(n=3)$, and wrestling $(n=1)$.

\subsection{Measures}

After receiving informed consent and demographic questions on gender, age and sport discipline, athletes were asked to state their main stressors, which could lead to a possible dropout in an open ended question format ("Do you have major chronic stressor(s) in your life as an athlete, which could lead to a dropout?"). Participants could indicate either "yes" or "no" in this dichotomous question format. In case of "no"-answers $(n=59)$, athletes were automatically treated as having no major stressor (NS). In case of "yes"-answers, athletes were asked to describe their major stressor. Therefore, athletes had the opportunity to state their stressors in short sentences or headwords. The "yes"-answers $(n=75)$ were used for qualitative analysis. For validation purpose, athletes were additionally asked to indicate the extent to which they had intentions of dropping out of competitive sports. Intentions were assessed using a 5-point rating scale $(1=$ never intended to drop out and $5=$ often intended to drop out).

\subsubsection{Depressive Symptoms}

The incidence of depressive symptoms in athletes was assessed with the widely used German version of the Center for Epidemiologic Studies Depression Scale (CES-D) from the National Institute of Mental Health (Hautzinger, Bailer, Hofmeister, \& Keller, 2011; Radloff, 1977). The CES-D is a short self-report scale designed to measure depressive symptomatology in the general population. The 20 items are assessed on a scale ranging from 0 to 3 . The cutoff for a major depressive episode is a score of $>22$. The scale has been found to have very high internal consistency ( $\alpha=0.89$ ), which was in the present study $\alpha=0.90$.

\subsubsection{Chronic Stress}

The Trier Inventory of Chronic Stress screening scale (Schulz, Schlotz, \& Becker, 2004) was used to identify athletes with a high level of chronic stress. The 12-item questionnaire measures five aspects of chronic stress: work overload, worries, social stress, lack of social recognition, excessive demands. The answers are provided on a 5 -point rating scale ranging from 0 - 4. Higher scores indicate a strong exposure to chronic stress. The scale was found to have a very high internal consistency $(\alpha=0.91$; Schulz et al., 2004). Reliability in the present study was also good with Cronbach's $\alpha=0.88$.

\subsection{Procedure}

After receiving informed consent, participants answered the online questionnaire containing these measures in predetermined order and therefore could not skip questions or go back to previous pages or questions. However, participants could quit the survey at any time. Before beginning the questionnaire, participants were provided

\footnotetext{
${ }^{1}$ Participants of the present study came from an online study in 2011 (Nixdorf et al., 2013). The goal of this study was to find prevalence rates and associations for depression in elite athletes. Within this online questionnaire the authors collected the present qualitative data on major stressors.
} 
with information about the study, including the estimated duration of the survey (30 min). The participants first answered questions related to demographic information, followed by completion of the questions covering the mentioned topics. At the end of the survey, contact details of the authors were provided.

\subsection{Data Analysis}

The open ended question on main chronic stressors was analyzed by a conventional content analysis (Hsieh \& Shannon, 2005). Therefore, categories were developed in an inductive process with an open coding, grouping and categorization and abstraction into a conceptual model (Elo \& Kyngäs, 2008). Sampling unit consisted of all stressors the participating athletes provided. Athletes were treated as recording units. Stressors were treated as content unit. For open coding, all content units were extracted, sighted and carefully read. In the second step, all content units were re-read and analyzed regarding overlapping, respectively similar, stressors. For grouping, all stressors were clustered into 17 subcategories (level b). In the next step, these 17 subcategories were grouped inductively into three main categories (level a), which had to be thematically fitting. Main categories were developed in order to gather different locations of main stressors. These three main categories were developed out of the data and carefully defined in the following step. Next, all athletes were rated into one of the three main categories according their stated main stressor by two raters. Rating was referred to the recording units in contrast to content units and therefore provided for each participant a categorization into one main category allowing a following analysis with distinct factor levels. Participants without any stressors provided were categorized into the category no stressor (NS). The inter-rater reliability was good with Cohen's $\kappa=0.86$. After the rating, stressors rated differently $(n=7)$ were discussed and categorized by both raters together.

Analyses of Variance (ANOVAs) were conducted for testing possible connections between the categories of stressors with chronic stress and depression. The stress-categories served as independent variables whereas depression, chronic stress and intentions of dropout served as dependent variables. As multiple ANOVAs were conducted, the problem of family wise error rate was accounted by setting alpha at $\alpha<0.01$. Post-hoc analyses of significant group differences within the independent variable were performed by Tukey's honest significance test (Tukey's HSD). In addition, effect sizes were calculated for significant differences. Validity of main stressors was tested by its connection to intentions of dropout. As the single-item question lacks psychometric values and therefore did not meet criteria for parametrical testing, Kruskal-Wallis One-Way Analysis of Variance was conducted. Following group differences were performed by a Wilcoxon Rank Sum Test, using pairwise comparisons with Bonferroni correction for adjusting alpha.

\section{Results}

The analysis of the open ended question (Table 1 ) resulted in 17 subcategories (level b) and three main categories (level a): Double burden (DB), sport specific demands (SSD) (physiological and psychological) and condi

Table 1. Structure and overview over the heading categories and subcategories.

\begin{tabular}{|c|c|}
\hline Categories, level a & Subcategories, level b \\
\hline Double Burden (DB) & $\begin{array}{l}\text { Education \& Job } \\
\text { Family \& Friends } \\
\text { (Free-) Time } \\
\text { Self-Determination }\end{array}$ \\
\hline $\begin{array}{c}\text { Sport Specific Demands (SSD) } \\
-\quad \text { physiological }\end{array}$ & $\begin{array}{c}\text { Bad performance } \\
\text { Injury } \\
\text { High training or competition load } \\
\text { Fatigue }\end{array}$ \\
\hline - psychological & $\begin{array}{l}\text { Pressure to perform } \\
\text { Mental stress } \\
\text { Fear of failure } \\
\text { Loss of joy } \\
\text { Motivational difficulties }\end{array}$ \\
\hline Conditions (C) & $\begin{array}{c}\text { Lack of perspective } \\
\text { Lack of sponsoring } \\
\text { Lack of support from an organizational level } \\
\text { Conflict within the team or with the trainer }\end{array}$ \\
\hline
\end{tabular}


tions $(C)$. The category DB was found to describe subcategories (level b), which illustrated stressors due to conflicting interests and responsibilities besides being an athlete. Demands such as educating themselves in school or university, having an own family or keeping up with other hobbies along with their main sports were considered as stressors in this category. Examples for this category are "not enough time for my family", "clash of training and school" or "no time for hobbies and friends". The category SSD represents physiological and psychological stressors in the context of sports and is directly linked to being an athlete. Physiological stressors were related to heavy exercise loads, injuries or bad performance levels, which is illustrated by examples like "not in shape for multiple weeks" or "multiple injuries". In the psychological aspect of the category, major stressors concerning psychological difficulties relating to high pressure to perform well, mental stress, fear of failure or motivational difficulties were named (i.e. "the pressure to always succeed is too much"). The category $\mathrm{C}$ represents stressors related to disadvantageous or unfavorable structures within the sport. Stressors like finances or problems with sponsoring, lack of support in the team or conflicts and lack of appreciation would fall into this category. Examples are "lack of support from my teammates" or "financial insecurities due to insufficient sponsoring”.

Results of the content analyses-ratings are displayed in Table 2, indicating equal distributions for the categories DB $(n=30)$ and SSD $(n=30)$ and fewer ratings in the category C $(n=15)$. In addition, the category NS was frequent with $n=59$. Results for the three ANOVAs for analyzing possible connections between the three main categories with depressive symptoms, chronic stress and intentions of dropout are shown in Table 2. Results show significant main effects across the four categories for depression $(F(3,130)=3.95 ; p<0.01)$, chronic stress $(F(3,130)=9.60 ; p<0.001)$, and intentions of dropout $\left(\chi^{2}(3)=74.19 ; p<0.001\right)$. Post-hoc analyses for intentions of dropout showed significant differences between the groups NS with DB, SSD and C (all $p<0.001$ ). For depression, significant differences were found between the groups NS and SSD $(p<0.01)$. Effect size for mean differences was moderate to strong with Cohens $\delta=0.78$. Regarding chronic stress, significant differences were found between the groups SSD and NS ( $p<0.001 ; \delta=1.15$ ), as well as tendencies between the groups NS and C $(p=0.016)$, and NS and DB $(p=0.049)$. No differences were found between the three stressor categories with respect to chronic stress, depressive symptoms or intentions of dropout.

\section{Discussion}

The present study was conducted to explore major stressors in elite athletes and its possible connection to depressive symptoms and chronic stress. Content analysis of open ended questions concerning major stressors led to three main categories: Double burden (DB) due to challenges of combining the career of an athlete with other duties, sport specific demands (SSD) such as heavy exercise loads, psychological pressure or failure, and conditions (C), which are referred to as stress through unfavorable structures within the team or organization. Further analyses revealed effects for chronic stress and depressive symptoms. Especially athletes with major stressors in the category SSD, representing psychological and physiological challenges in the context of sport, were found to have higher scores in depression and chronic stress. Firstly, the results of the content analysis and secondly, the found effects for chronic stress and depression will be discussed in the following.

Table 2. Results from content analysis rating for sample size, means and ANOVA for chronic stress, depression and intetions of dropout.

\begin{tabular}{|c|c|c|c|c|c|c|}
\hline & \multicolumn{5}{|c|}{ Categories } & \multirow{2}{*}{$\begin{array}{c}\text { ANOVA } \\
F / \chi^{2} \text {-value and degrees } \\
\text { of freedom }(d f)\end{array}$} \\
\hline & $\mathrm{DB}$ & SSD & $\mathrm{C}$ & NS & Overall & \\
\hline Sample Size $n$ & $n=30$ & $n=30$ & $n=15$ & $n=59$ & $N=134$ & - \\
\hline Chronic stress & $M=17.8$ & $M=22.8$ & $M=20.5$ & $M=12.4$ & $M=16.8$ & $F(3,130)=9.60^{* *}$ \\
\hline Depression & $M=14.0$ & $M=16.9$ & $M=13.5$ & $M=10.3$ & $M=12.9$ & $F(3,130)=3.95^{*}$ \\
\hline Dropout intentions & $M=2.8$ & $M=2.7$ & $M=3.0$ & $M=1.4$ & $M=2.2$ & $\chi^{2}(3)=74.19^{* *}$ \\
\hline
\end{tabular}

Note: Categories abbreviations DB = Double Burden, SSD = Sport Specific Demands, C = Conditions, NS = No Stressor. ${ }^{* *} p<0.001,{ }^{*} p<0.01$. 


\subsection{Major Stressors in Elite Athletes}

The process of abstraction throughout content analysis led to a further characterization of the three found categories. By describing the categories throughout location of the inherent stressors, SSD are located within the athlete (intra athletic). Stressors within this category are directly linked to possible physiological and psychological issues athletes have to deal with. In contrast, the category $\mathrm{C}$ is related to stressors located in the direct surrounding of athletes (e.g. in their teams, organization). Furthermore, the category DB represents stressors which result from the private life or through the conflict between the multiple roles of an athlete. Therefore, stressors in this category can be considered stressors with relation to the whole life of a person and are not merely bound to athletic or sports related aspects.

A categorization of stressors by Hanton et al. (2005) led to a differentiation between competitive and organizational stressors. The authors concluded that athletes experience and recall more demands related to competition. As the present results indicate few stressors in the category $\mathrm{C}$ and more in the category SSD one might draw equal conclusions. However, in this study athletes provided also several stressors regarding conflicts between sports and private life (DB) which are clearly not competition related. Other comparable classifications have their origin in research on dropout. Enoksen (2011) provides an overview about several causes of dropouts in the literature, including (1) training and performance factors; (2) education and work obligations; (3) motivational aspects; (4) social environment and (5) choice of other sport activities and interests. The author in particular underlines reasons due to injuries, performance stagnation, educational demands and lack of motivation as highly relevant factors (Enoksen, 2011). Similarities with the category DB especially appear regarding the second factor: education and work obligations. This is not surprising, as the major stressors in this study were linked to intentions of dropout. In addition, goal of the study was to link major stressors with chronic stress and depressive symptoms. Therefore, comparisons with other conceptual frameworks and categories are difficult and should be interpreted carefully.

\subsection{Stressors, Chronic Stress and Depressive Symptoms}

Regarding levels of chronic stress, effects were found for the category SSD along with tendencies for both other categories. This indicates that the stated stressors are associated with experiences of chronic stress among elite athletes. This effect validates the open ended question, as the study's goal was to explore main stressor with a chronic character. In detail, especially stressors within the athlete (psychological and physiological) are connected to higher levels of chronic stress. This effect was also seen for depressive symptoms. Here, the category SSD also accounted for the effect between major stressors and depressive symptoms with a moderate to strong effect size. Taken together, the present results indicate that mainly stressors linked to sport specific psychological and physiological demands of an athlete are related to negative experiences like chronic stress or depressive symptoms.

The present results clearly do not allow underestimating other, more sport distal stressors in an athlete's life. However, considering psychological aspects of the three categories might lead to testable assumptions for further research on this topic. One such possible psychological assumption in this regard lies in the different location of stressors within the three major categories. SSD, for example, represent stressors directly linked to athletes. Therefore, stressors within this category might have an impact on important aspects of an athlete's identity. Following this assumption, stressors within the category $\mathrm{C}$ might be relevant for athletes, too. However, as they are not directly involved, athletes have the opportunity to make other persons or circumstances accountable for the current stress. Concerning the category DB, one might expect an equally heavy impact on athletes, as aspects like education, relationship or family seem also highly important. However, having various aspects in one's life offers also the opportunity to have reinforcements by others. Therefore, difficulties in one aspect could be compensated throughout positive other aspects.

Other findings on specific events in an athlete's life such as injury (Appaneal et al., 2009) or failure (Hammond, Gialloreto, Kubas, \& Davis, 2013) clearly indicate towards the importance of sport specific mechanisms for depression in athletes. However, not only single events but chronic stressors should be considered following the present results. Therefore, chronic stress and stressors seem to play an important role. Nevertheless, episodes like injuries, failure or overtraining might have an even longer and therefore chronic impact on an athlete's health than the event itself. To answer this question research would need to evaluate long-term effects of such 
factors.

The found effects in the present study regarding the location of important stressors for athletes can be viewed from a practical perspective, too. Here, the specific aspects of sports in sport psychological counseling and psychotherapy can be taken into consideration. The results indicate that clearly sport related challenges (e.g. overwhelming demands of training, pressure to perform well) are connected to stress and depression. Unfortunately, little is known on clinical issues in elite athletes such as depression (Wolanin, Gross, \& Hong, 2015) and therefore on sport specific mechanisms in development and therapy of psychological disorders. Thus, practitioners in the field of clinical sport psychology (sport psychologists and therapists) would benefit of a better understanding in this intersection.

\subsection{Limitations}

The present study is explorative in its nature and therefore does not test specific assumptions based on theories. Consequently, all results cannot be seen as empirical evidences and should rather be regarded as insight, which further research can base testable assumptions on. The found major stressor categories have to be interpreted with care, as they were developed inductively by qualitative research methods. Furthermore, findings are constrained by the research design. Sample characteristics such as size, nationality, or sport discipline can limit external validity of the present results. Furthermore, causal implications cannot be drawn by the explorative, cross-sectional design of the study. However, following research on chronic stress or depression in athletes could possibly benefit by considering sport related challenges and stressors and therefore conduct studies to test causal assumptions. In addition, this study focused merely on negative outcomes and therefore on possible negative influences (stressors). As discussed above, there also might be positive aspects related with the found stressful topics for elite athletes (e.g. reinforcement by family or school). Furthermore, other individual resources (e.g. support system, self-confidence) are possibly influencing experiences of stress and depression in a positive manner. Although not explored in the present study, such aspects might be valuable for further research.

\section{Conclusion}

The present study explored major stressors in elite athletes and found three main categories for sources of stress: double burden, sport specific demands and conditions. Major stressors seemed to be located in various, different aspects of an athlete's life. Thus, athletes may face different challenges during their careers and stress may origin from the daily training, the interaction with coaches and teammate, but also from conflicting interests regarding the career and private life. Further analysis showed connections between the stressors and experiences of chronic stress as well as with depressive syndromes in elite athletes. Especially stressors related to sport specific demands seemed accountable for this effect. Therefore, further knowledge on sport specific mechanisms regarding outcomes such as depression and stress is needed. Research on depression and other general psychological health issues could possibly benefit by taking such sport specific aspects (e.g. performance pressure, exercise loads, etc.) into greater consideration.

\section{References}

Angeli, A., Minetto, M., Dovio, A., \& Paccotti, P. (2004). The Overtraining Syndrome in Athletes: A Stress-Related Disorder. Journal of Endocrinological Investigation, 27, 603-612. http://dx.doi.org/10.1007/BF03347487

Appaneal, R. N., Levine, B. R., Perna, F. M., \& Roh, J. L. (2009). Measuring Post Injury Depression among Male and Female Competitive Athletes. Journal of Sport and Exercise Psychology, 31, 60-76.

Armstrong, L. E., \& VanHeest, J. L. (2002). The Unknown Mechanism of the Overtraining Syndrome: Clues from Depression and Psychoneuroimmunology. Sports Medicine, 32, 185-209.

http://dx.doi.org/10.2165/00007256-200232030-00003

Crocker, P. R. E, \& Graham, T. R. (1995). Coping by Competitive Athletes with Performance Stress: Gender Differences and Relationships with Affect. The Sport Psychologist, 9, 325-338.

Elo, S., \& Kyngäs, H. (2008). The Qualitative Content Analysis Process. Journal of Advanced Nursing, 62, 107-115. http://dx.doi.org/10.1111/j.1365-2648.2007.04569.x

Enoksen, E. (2011). Drop-Out Rate and Drop-Out Reasons among Promising Norwegian Track and Field Athletes: A 25 Years Study. Scandinavian Sport Studies Forum, 2, 19-43. 
Fletcher, D., Hanton, S., Mellalieu, S. D., \& Neil, R. (2012). A Conceptual Framework of Organizational Stressors in Sport Performers. Scandinavian Journal of Medicine \& Science in Sports, 22, 545-557. http://dx.doi.org/10.1111/j.1600-0838.2010.01242.x

Frank, R., Nixdorf, I., \& Beckmann, J. (2013). Depression in Elite Athletes: Prevalence and Psychological Factors. Deutsche Zeitschrift für Sportmedizin [German Journal of Sports Medicine], 64, 320-326.

Gould, D., Jackson, S., \& Finch, L. (1993). Sources of Stress in National Champion Figure Skaters. Journal of Sport and Exercise Psychology, 15, 134-159.

Hammen, C., Kim, E. Y., Eberhart, N. K., \& Brennan, P. A. (2009). Chronic and Acute Stress and the Prediction of Major Depression in Women. Depression and Anxiety, 26, 718-723. http://dx.doi.org/10.1002/da.20571

Hammond, T., Gialloreto, C., Kubas, H., \& Davis, H. H. (2013). The Prevalence of Failure-Based Depression among Elite Athletes. Clinical Journal of Sport Medicine, 23, 273-277. http://dx.doi.org/10.1097/JSM.0b013e318287b870

Hanton, S., Fletcher, D., \& Coughlan, G. (2005). Stress in Elite Sport Performers: A Comparative Study of Competitive and Organizational Stressors. Journal of Sports Sciences, 23, 1129-1141. http://dx.doi.org/10.1080/02640410500131480

Hautzinger, M., Bailer, M., Hofmeister, D., \& Keller, F. (2011). Allgemeine Depressionsskala (ADS) [German Version of the CES-D Scale] (2nd ed.). Göttingen: Hogrefe.

Hsieh, H. F., \& Shannon, S. E. (2005). Three Approaches to Qualitative Content Analysis. Qualitative Health Research, 15, 1277-1288. http://dx.doi.org/10.1177/1049732305276687

Kellmann, M. (2010). Preventing Overtraining in Athletes in High-Intensity Sports and Stress/Recovery Monitoring. Scandinavian Journal of Medicine \& Science in Sports, 20, 95-102. http://dx.doi.org/10.1111/j.1600-0838.2010.01192.x

Leddy, M. H., Lambert, M. J., \& Ogles, B. M. (1994). Psychological Consequences of Athletic Injury among High-Level Competitors. Research Quarterly for Exercise and Sport, 65, 347-54. http://dx.doi.org/10.1080/02701367.1994.10607639

Lee, S., Jeong, J., Kwak, Y., \& Park, S. K. (2010). Depression Research: Where Are We Now? Molecular brain, $3,8$. http://dx.doi.org/10.1186/1756-6606-3-8

Lehmann, M., Foster, C., Gastmann, U., Keizer, H., \& Steinacker, J. M. (1999). Definition, Types, Symptoms, Findings, Underlying Mechanisms, and Frequency of Overtraining and Overtraining Syndrome. In M. Lehmann, C. Foster, U. Hastmann, H. Keizer, \& J. M. Steinacker (Eds.), Overload, Performance Incompetence, and Regeneration in Sport (pp. 1-6). US: Springer. http://dx.doi.org/10.1007/978-0-585-34048-7_1

Lemyre, P.-N., Roberts, G. C., \& Stray-Gundersen, J. (2007). Motivation, Overtraining and Burnout: Can Self-Determined Motivation Predict Overtraining and Burnout in Elite Athletes. European Journal of Sport Sciences, 7, 115-132. http://dx.doi.org/10.1080/17461390701302607

Meeusen, R., Duclos, M., Foster, C., Fry, A., Gleeson, M., Nieman, D., \& Urhausen, A. (2013). Prevention, Diagnosis, and Treatment of the Overtraining Syndrome: Joint Consensus Statement of the European College of Sport Science and the American College of Sports Medicine. Medicine and Science in Sports and Exercise, 45, 186-205. http://dx.doi.org/10.1249/MSS.0b013e318279a10a

Monroe, S. M., \& Reid, M. W. (2009). Life Stress and Major Depression. Current Directions in Psychological Science, 18, 68-72. http://dx.doi.org/10.1111/j.1467-8721.2009.01611.x

Nixdorf, I., Frank, R., Hautzinger, M., \& Beckmann, J. (2013). Prevalence of Depressive Symptoms and Correlating Variables among German Elite Athletes. Journal of Clinical Sport Psychology, 7, 313-326.

Noblet, A. J., \& Gifford, S. M. (2002). The Sources of Stress Experienced by Professional Australian Footballers. Journal of Applied Sport Psychology, 14, 1-13. http://dx.doi.org/10.1080/10413200209339007

O’Connor, P. J., Morgan, W. P., Raglin, J. S., Barksdale, C. M., \& Kalin, N. H. (1989). Mood State and Salivary Cortisol Levels Following Overtraining in Female Swimmers. Psychoneuroendocrinology, 14, 303-310. http://dx.doi.org/10.1016/0306-4530(89)90032-2

Puffer, J. C., \& McShane, J. M. (1992). Depression and Chronic Fatigue in Athletes. Clinics in Sports Medicine, 11, 327338.

Radloff, L. S. (1977). The CES-D Scale: A Self-Report Depression Scale for Research in the General Population. Applied Psychological Measurement, 1, 385-401. http://dx.doi.org/10.1177/014662167700100306

Schaal, K., Tafflet, M., Nassif, H., Thibault, V., Pichard, C., Alcotte, M., \& Toussaint, J.-F. (2011). Psychological Balance in High Level Athletes: Gender-Based Differences and Sport-Specific Patterns. PLoS ONE, 6, e:19007.

Schulz, P., Schlotz, W., \& Becker, P. (2004). Trierer Inventar zum chronischen Stress (TICS) [Trierer Inventory of Chronic Stress]. Göttingen: Hogrefe.

Wolanin, A., Gross, M., \& Hong, E. (2015). Depression in Athletes: Prevalence and Risk Factors. Current Sports Medicine Reports, 14, 56-60. http://dx.doi.org/10.1249/JSR.0000000000000123 